\title{
Power-to-Gas Pathways to a Fossil Free Energy System
}

\author{
Michael Fowler \\ University of Waterloo \\ Department of Chemical Engineering \\ Waterloo, Ontario, Canada
}

Power-to-gas is a novel energy storage concept that can help in providing energy storage and offer a sustainable and efficient alternative ways utilize the surplus electricity generated by the provincial grid of Ontario, Canada. The ability of the power to gas energy hubs to utilize the existing natural gas distribution and storage network (within the province) to distribute and store the electrolytic hydrogen produced is one of its major advantages. There a number of different 'pathway' for using the hydrogen generated via the Power-to-Gas concept, and this presentation will outline the pathways for use of the hydrogen and for the provision of energy storage. Also an optimization model of a power to gas energy hub having a hydrogen production module capacity of $2 \mathrm{MW}$ has been developed and will be presented. The goal of this optimization study was to carry out an economic feasibility of the energy hub under existing pricing mechanisms for the three primary services that it provides, namely: 1) Offsetting $\mathrm{CO} 2$ emissions at natural gas end users by providing hydrogen enriched natural gas; 2) Providing demand response when directed by the Independent Electricity System Operator of the province, and 3) Providing pure hydrogen to a fuel cell vehicle refueling station. 